\title{
Interference spectroscopy with coherent anti-Stokes Raman scattering of noisy broadband pulses.
}

\author{
Evgeny A. Shapiro ${ }^{1}$, Stanislav O. Konorov ${ }^{1}$, Valery Milner ${ }^{2}$, \\ Departments of Chemistry ${ }^{1}$ and Physics ${ }^{2}$, The University of British Columbia \\ 2036 Main Mall, Vancouver, BC, Canada V6T $1 Z 1$
}

(Dated: September 2, 2021)

\begin{abstract}
We propose a new technique for comparing two Raman active samples. The method employs optical interference of the signals generated via coherent anti-Stokes Raman scattering (CARS) of broadband laser pulses with noisy spectra. It does not require spectrally resolved detection, and no prior knowledge about either the Raman spectrum of the samples, or the spectrum of the incident light is needed. We study the proposed method theoretically, and demonstrate it in a proof-of-principle experiment on Toluene and ortho-Xylene samples.
\end{abstract}

\section{INTRODUCTION.}

In the last decade, coherent nonlinear optical spectroscopy with femtosecond pulses has evolved into a powerful tool for chemical characterization, detection, and microscopy [1-8]. Ultrashort laser pulses exhibit high peak intensities, which result in high nonlinear signals, at low average power below the damage threshold of many systems of interest. Coherent anti-Stokes Raman scattering (CARS) with time-resolved (tr) and frequency-resolved (fr) detection has been recently complemented by a number of methods employing shaped femtosecond pulses. By adjusting the amplitudes and phases of the spectral components of a broadband pulse, one is able to enhance Raman excitation of one molecular species while not exciting another, thus gaining sensitivity to chemical structure [9]. Pulse shaping also proved useful in suppressing the non-resonance background signal which usually reduces CARS sensitivity [10-13]. Pulse shaping approaches to coherent nonlinear spectroscopy typically rely on the availability of broadband pulses with smooth well-characterized spectral and temporal profiles [14].

In many situations, the goal of the spectroscopic analysis is a quick "yes" or "no" answer to the question of whether the two samples of interest are similar. One example is a quality 
control task in which the sample in question is compared with a reference. Here, it is desirable to avoid experiments which require time-consuming scanning procedures, e.g. delay scanning in tr-CARS or frequency scanning in fr-CARS detection. Single-shot techniques based on femtosecond pulse shaping, although very quick, are often sensitive to the a priori knowledge about the anticipated spectral response from the sample of interest. Another quick scanless approach to molecular analysis known as multiplex CARS (broadband excitation with narrowband probing) relies on spectrally resolved detection and analysis of the signal.

In this paper we propose and demonstrate a technique for the direct spectroscopic comparison of two Raman active samples. In the following text we refer to them as "reference" (" $R$ ") and "sample" (" $S$ "), and imply that the goal of the experiment is to establish the degree of their similarity. The proposed method does not require time or frequency scanning, and can be implemented without technically involved pulse shaping. Spectral analysis of the signal is not required; rather, an integral power of a single anti-Stokes beam is detected. The scheme does not rely on the a priori knowledge of Raman spectra of either the sample of interest or the reference. Moreover, it requires neither characterization nor control over the spectrum of the input beams. One can employ probe pulses with unknown random spectral profiles, as long as the latter exhibit sufficiently narrow features. One example of such spectrum is that of a transform-limited pulse sent through a random scatterer.

\section{METHOD}

The idea of the method is shown in Fig.1(a). Laser pulses pass through the reference and sample - illustrated in the Figure by two glasses of wine, - generating a nonlinear spectroscopic response in both of them. If the two materials are similar (different), their spectroscopic responses are similar (different) as well. Varying the phase $\Phi$ added to the nonlinear optical signal between the two media, one gains $100 \%$ interference contrast in the case of identical samples (" $S=R$ "), and a lower contrast if $S$ and $R$ differ (" $S \neq R$ "). As shown below, the interference contrast is a natural measure of the similarity between the $S$ and $R$ spectra.

We implement the idea described above using femtosecond CARS spectroscopy. In femtosecond CARS, broadband pump and Stokes laser pulses excite a long-lived Raman coherence in the medium. A probe pulse, which can be either narrow- or broadband, stimulates 

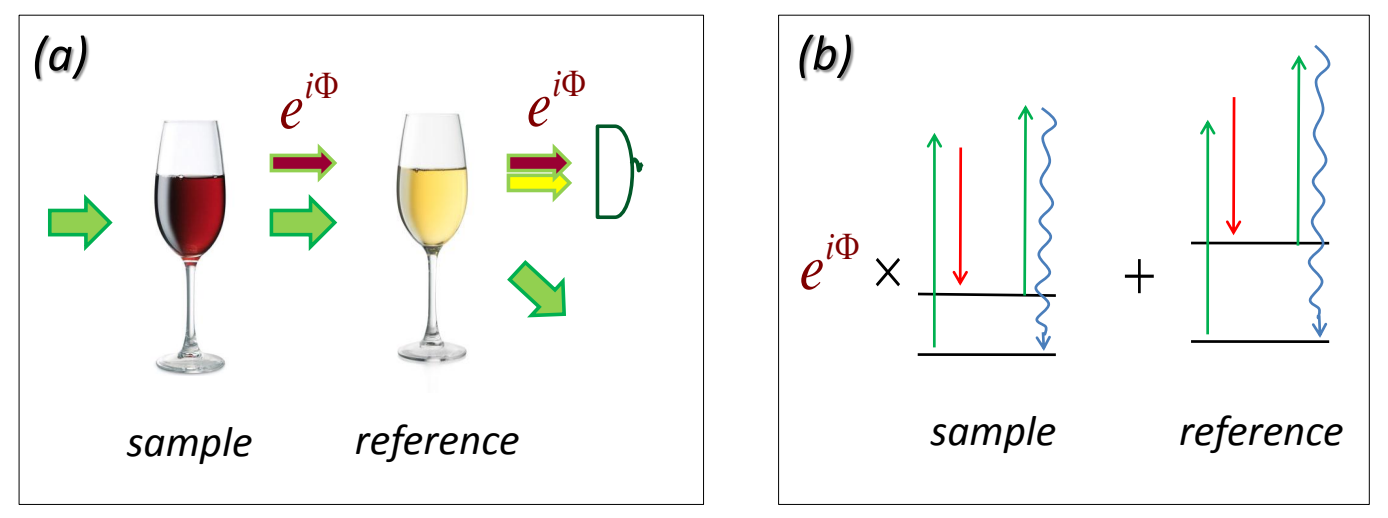

FIG. 1: Color online. The idea of the method. (a): Two nonlinear signals (thin red and yellow arrows) are generated in two media of interest, Sample and Reference, and interfere at the detector. Variable phase shift $\Phi$ is added to one of the signals. Interference is strongest when the sample and the reference are similar. (b): Simplified energy-level diagram for the proposed method. Similar samples generate ansi-Stokes radiation (curly blue lines) at the same frequency, leading to strong interference. Chemically different samples generate signal at different frequencies, leading to no interference.

radiation at the anti-Stokes frequency $\omega_{A S}=\omega_{p}-\omega_{S}+\omega_{p r}$, where $\omega_{p}, \omega_{S}, \omega_{p r}$ are the frequencies of the pump, Stokes, and probe spectra, respectively. The anti-Stokes signal consists of two parts. The resonant signal, whose time duration is determined by the life times of the Raman modes, is given by [15-17]

$$
E_{r}(\omega)=\int_{-\infty}^{-\infty} d \Omega \sum_{n} C_{n} E(\omega-\Omega) A(\Omega) \frac{1}{\Omega-\Omega_{n}+i \Gamma_{n}} .
$$

Here $n$ enumerates Raman resonances (e.g. vibrational energy levels of the molecule), $\Omega_{n}$ are their energies, $\Gamma_{n}$ are their widths,

$$
A(\Omega)=\int_{-\infty}^{\infty} d \omega^{\prime} E^{*}\left(\omega^{\prime}-\Omega\right) E\left(\omega^{\prime}\right)
$$

is the two-photon excitation spectrum, and $E(\omega)$ is the spectral amplitude of the joint pump, Stokes, and probe field. The non-resonant background (NRB) signal, corresponding to the instantaneous electronic responce, can be approximated as

$$
E_{n r}(\omega)=C_{\text {nonres }} \int_{-\infty}^{\infty} E(\omega-\Omega) A(\Omega) d \Omega
$$

and can be either weaker or stronger than the resonant one. In order to separate $E_{r}$ from $E_{n r}$, one can delay probe pulses with respect to pump and Stokes pulses, as described below. 
Consider, first, a model situation in which both $R$ and $S$ Raman spectra consist of a single line of width $\Gamma$, centered at $\Omega_{R}$ and $\Omega_{S}$, respectively (Fig.1(b)). The idea of our method is most transparent in the multiplex CARS scheme, where a broadband pump-Stokes excitation is followed by a narrowband probe. For the latter, we assume that $E_{p r}(\omega)=E_{0}$ within a narrow interval $\delta \omega$ near the probe frequency $\omega_{p r}$, and $E_{p r}(\omega)=0$ otherwise. By our design, the CARS signal acquires an extra phase factor $e^{i \Phi}$ after exiting the sample medium. Assuming for simplicity that the broadband excitation is uniform, i.e. $A(\Omega)=A_{0}$ in the relevant frequency range of the excited Raman modes, we obtain from Eq.(1) the following resonant signal generated in both materials:

$$
E_{r}(\omega)=\delta \omega E_{0} A_{0}\left[\frac{e^{i \Phi} C_{S}}{\omega-\omega_{p r}-\Omega_{S}+i \Gamma}+\frac{C_{R}}{\omega-\omega_{p r}-\Omega_{R}+i \Gamma}\right] .
$$

CARS signal from the sample is in the vicinity of $\omega_{C A R S}^{S}=\Omega_{S}+\omega_{p r}$ while for the reference it is near $\omega_{C A R S}^{R}=\Omega_{R}+\omega_{p r}$. When the phase $\Phi$ is scanned, these signals will interfere if $\Omega_{S}=\Omega_{R}$, and will not interfere otherwise (Fig.2). To quantify this interference, we calculate the integral signal by integrating $E_{r}(\omega) E_{r}^{*}(\omega)$ over all frequencies. Introducing the dimensionless frequency $w=\left(\omega-\omega_{p r}\right) / \Gamma$, we have from Eq.(4)

$$
\left\langle E_{r} E_{r}^{*}\right\rangle_{\omega}(\Phi)=\delta \omega^{2}\left|E_{0} A\right|^{2} \int_{-\infty}^{\infty} d w\left|\frac{e^{i \Phi} C_{S}}{w-w_{S}+i}+\frac{C_{R}}{w-w_{R}+i}\right|^{2}
$$

where $w_{R, S}=\Omega_{R, S} / \Gamma$, and \langle\rangle$_{\omega}$ stands for the integration over frequency. The integral in Eq.(5) is readily taken by contour integration, which yields

$$
\left\langle E_{r} E_{r}^{*}\right\rangle_{\omega}(\Phi)=N_{\text {multiplex }} \times\left[\left|C_{S}\right|^{2}+\left|C_{R}\right|^{2}+\frac{\left|C_{S} C_{R}\right|}{1+w_{R S}^{2}} \cos \left[\Phi-\Delta-\phi_{C}\right]\right]
$$

where

$$
\begin{aligned}
N_{\text {multiplex }} & =2 \pi \delta \omega^{2}\left|E_{0} A\right|^{2} \\
w_{R S} & =\frac{\Omega_{S}-\Omega_{R}}{2 \Gamma} \\
\tan \Delta & =w_{R S} \\
\phi_{C} & =\arg \left[C_{S}^{*} C_{R}\right] .
\end{aligned}
$$

Equation (6) is the key for understanding the proposed method. If $S$ and $R$ are similar, then $w_{R S} \approx 0$, and the interference contrast in the integral signal is $100 \%$ : at $\Phi_{\min }=\pi$ the resonant CARS intensity drops to zero, as seen in Fig.2(a,c). If the two samples are 

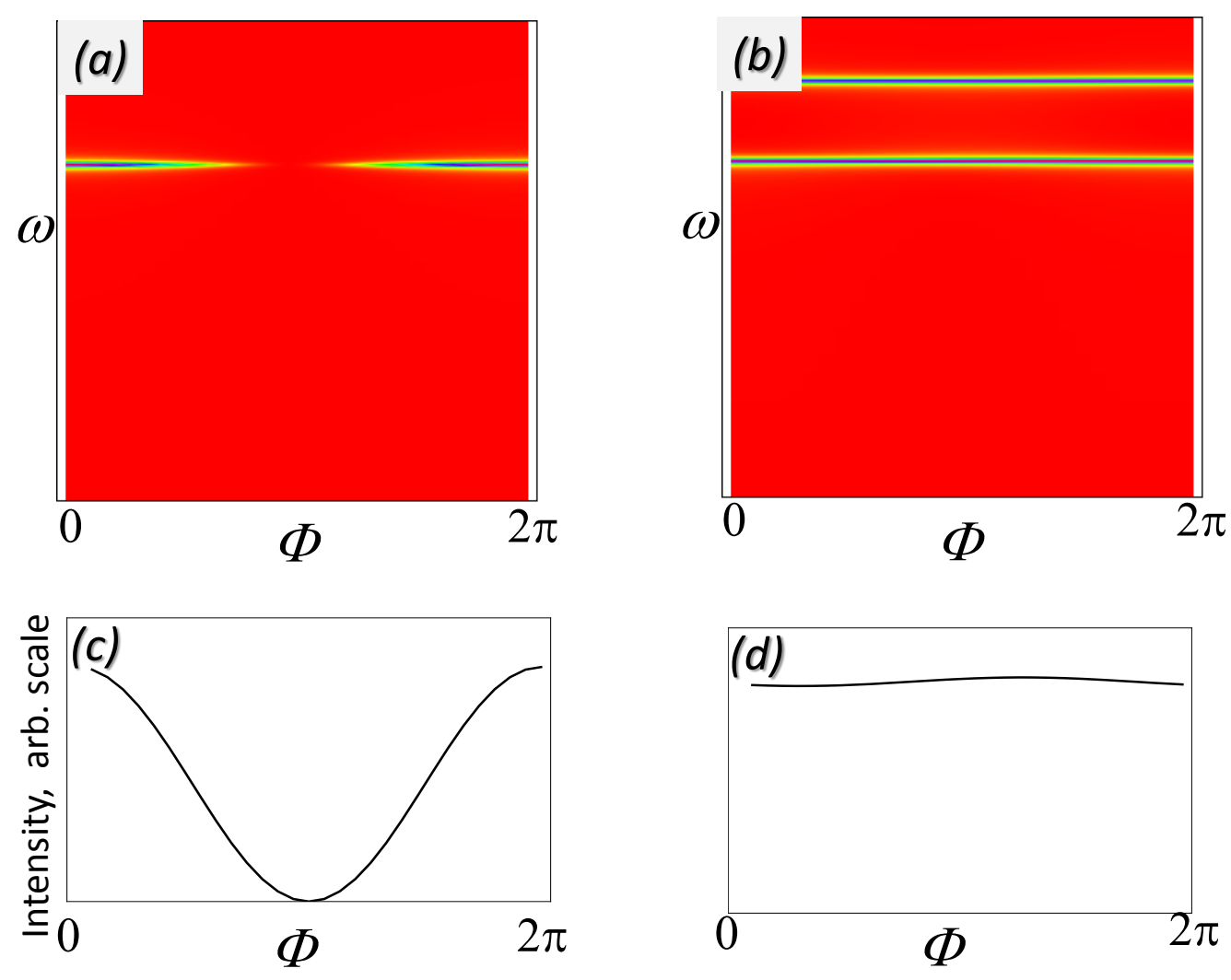

FIG. 2: Color online. (a,b): Two-dimensional interference maps for the intensity of multiplex CARS probing two similar (a) and different (b) samples. (c,d): CARS signals integrated over frequency.

completely different, the corresponding CARS signals are at two different frequencies, and therefore do not interfere - this is the situation shown in Fig.2(b,d). Finally, if $S$ and $R$ lines partially overlap, the integral interference pattern is different from that of two similar samples in two ways. First, its contrast is lower. If the $S$ and $R$ signals are of the same strength, $C_{S}=C_{R}$, then the contrast is reduced due the factor $1 /\left(1+w_{R S}^{2}\right)$ in the square brackets of Eq.(6). From Eq.(8), one can see that $w_{R S}$ may indeed be viewed as a natural measure of the mismatch between the two Raman spectra. Second, the minimum and maximum of the integral signal $\left\langle E_{r} E_{r}^{*}\right\rangle_{\omega}(\Phi)$ are offset from their values $\Phi_{\text {min }}=\pi$ and $\Phi_{\max }=0$ by the value $\Delta+\phi_{C}$. This situation is illustrated in Figs. 2 and 3.

We now show that having a single narrowband probe pulse (as in multiplex CARS) is not essential for the proposed interference detection. Let us illuminate the $S$ and $R$ media with transform-limited broadband pump and Stokes pulses, and a probe pulse that consists 
of $N$ narrowband components with an average spectral width $\delta \omega$. The spectral separation between these components is not important: one may employ a set of well isolated lines, as well as a single broadband line with a random phase modulation under its spectral envelope. The only relevant parameter is the spectral correlation length $\delta \omega$ of the complex probe field. It is important, however, that the phases of $N$ probe's spectral components are not correlated with each other.

The resonant anti-Stokes response now has the form

$$
E_{r}(\omega)=\delta \omega \sum_{i=1}^{N} E_{i} A\left(\omega-\omega_{p r i}\right)\left[\frac{e^{i \Phi} C_{S}}{\omega-\omega_{p r i}-\Omega_{S}+i \Gamma}+\frac{C_{R}}{\omega-\omega_{p r i}-\Omega_{R}+i \Gamma}\right]
$$

This equation is similar to Eq.(4), except that the single term $E_{0} A_{0}$ is replaced by a sum of $N$ complex amplitudes $E_{i}=\left|E_{i}\right| \exp \left[i \phi_{i}\right]$ multiplied by the Raman excitation amplitudes $A\left(\omega-\omega_{\text {pri }}\right)$. The intensity of the CARS signal $E_{r}(\omega) E_{r}^{*}(\omega)$ thus contains $N^{2}$ terms,

$$
\begin{aligned}
E_{r}(\omega) E_{r}^{*}(\omega)= & \delta \omega^{2} \sum_{i, j}\left|E_{i} E_{j}\right| e^{i\left(\phi_{i}-\phi_{j}\right)} A\left(\omega-\omega_{p r i}\right) A^{*}\left(\omega-\omega_{p r j}\right) \\
& \times\left[\frac{e^{i \Phi} C_{S}}{\omega-\omega_{p r i}-\Omega_{S}+i \Gamma}+\frac{C_{R}}{\omega-\omega_{p r i}-\Omega_{R}+i \Gamma}\right] \\
& \times\left[\frac{e^{-i \Phi} C_{S}^{*}}{\omega-\omega_{p r j}-\Omega_{S}-i \Gamma}+\frac{C_{R}^{*}}{\omega-\omega_{p r j}-\Omega_{R}-i \Gamma}\right] .
\end{aligned}
$$

Within a given realization of a random probe spectrum, the interference pattern in the $(\omega, \Phi)$ plane can be quite complex. CARS signal consists of many spectral lines, reflecting both the $S$ and $R$ Raman resonances convolved with the probe spectrum. Two examples are shown in Fig.3. If the $S=R$, the two terms in the square brackets of Eq.(11) are similar, and the interference contrast at each frequency is high (Fig.3(a)). However, if the two materials are different, the cross terms in Eq.(12) contribute to the interference pattern in a random uncorrelated way. Depending on the spectral amplitudes $\left|E_{i}\right|$ and phase differences $\phi_{i}-\phi_{j}$, the interference fringes at different frequencies lose their contrast and cease to occur at the same values of $\Phi$. As a result, the intensity map in the $(\omega, \Phi)$ plane shows no regular fringe structure, as demonstrated in Fig.3(b).

The apparent randomness of the CARS intensity map disappears once it is averaged over several noise realizations. Since the phases $\phi_{i}$ of different spectral components of the probe field are not correlated, the cross terms in Eq.(12) average out, while those with $i=j$ do not. The resulting expression is similar to Eq.(5), but with a normalization factor accounting for 

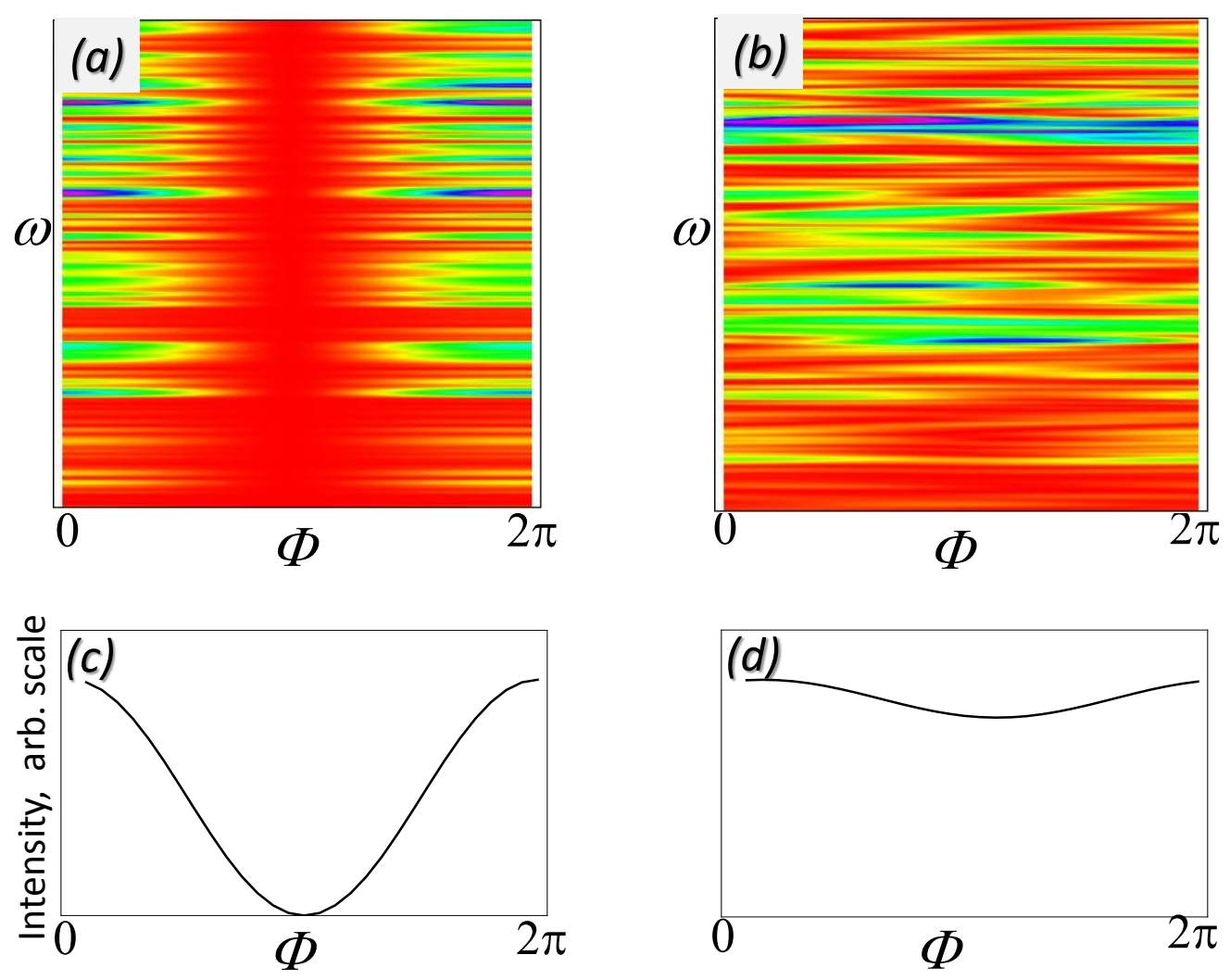

FIG. 3: Color online. (a,b): Simulated interference map for CARS with noisy probe with (a): two Toluene samples, and (b): Toluene and ortho-Xylene samples. (c,d): CARS interference with a noisy probe, averaged over noise realizations and integrated over frequency. (c): two Toluene samples; (d): Toluene and ortho-Xylene samples.

having $N$ spectral lines in the probe. By repeating the steps which lead to Equation (6), we obtain, for the case of a noisy probe pulse, an expression of exactly the same form. The only difference is the normalization constant $N_{\text {noise }}$ :

$$
N_{\text {noise }}=2 \pi \delta \omega^{2}\left|A_{0}\right|^{2} \sum_{i=1}^{N}\left\langle\left|E_{p r, i}\right|^{2}\right\rangle
$$

where $\langle.$.$\rangle denotes averaging over noise realizations, and A_{0}$ is the Raman excitation probability, which is assumed to be uniform in the relevant frequency range. If all the spectral lines of the probe pulse are of the same intensity, the integrated CARS intensity is simply $N$ times that generated by a single spectral component.

If broadband pump and Stokes pulses excite several vibrational resonances, one has to add in Eq.(6) a sum over all $S$ and $R$ lines. For different substances, the lines will, in general, 
be randomly placed with respect to each other. The value $w_{R S}$ (Eq.8) will be positive for some pairs of lines and negative for others. After summation over $\Omega_{S}$ and $\Omega_{R}$, the minima and maxima of the resulting interference pattern may not shift much from $\Phi_{\min }=\pi$ and $\Phi_{\max }=0$. Yet the contrast of the interference pattern will remain low if the $S$ and $R$ materials differ significantly. The contrast depends on the average value of $w_{R S}-$ a natural measure of the similarity of the two spectra.

We numerically simulated an interference of two noisy CARS signals from Toluene $\left(\mathrm{C}_{7} \mathrm{H}_{8}\right)$ and o-Xylene $\left(\mathrm{C}_{8} \mathrm{H}_{10}\right)$, whose Raman spectra are well known. The excitation pulse parameters were similar to those used in our experiments and described in Sec.III. Fig.3(a,b) shows two calculated interference patterns. In plot (a), Toluene was used as both the Sample and Reference, whereas replacing the Sample by o-Xylene resulted in plot (b). Integration over frequency produced the results which are plotted in panels $(c, d)$ of the Figure and demonstrate a striking difference in the interference contrast for the cases of Toluene-Toluene and Toluene- - -Xylene interference. Within a wide set of parameters, the simulations showed a $100 \%$ fringe visibility for the interference of two identical substances, and only $\sim 17 \%$ visi-

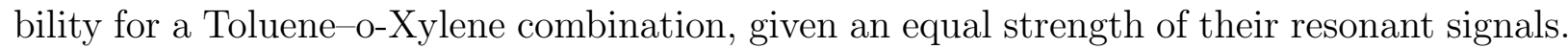
The $\sim 17 \%$ visibility of the Toluene-o-Xylene interference is due to the partial overlap of their Raman spectral lines, and thus gives the measure of their spectral similarity in accord with Eq.(6).

Our numerical tests allowed us to make several observations. First, the integrated picture is insensitive to the spectral shapes of pump, Stokes, and probe pulses as long as the correlation length of noise introduced to the probe spectrum is smaller than the average separation between the $S$ and $R$ Raman lines. Second, having many independent lines in the probe spectrum is advantageous as compared to having a few lines. In the case of many lines, not only the signal is stronger (see Eq.(13)), but one also needs to average the interference map over fewer noise realizations. Finally, we noticed that using random pulses for the excitation, as well as for probing, leads to qualitatively similar results, although requires averaging over large numbers of noise realizations, and gives higher interference contrasts for the " $S \neq R$ " case. Assuming non-equal signal strengths further reduced the interference visibility, enabling an easy distinction between the similar-samples and different-samples combinations.

As expected and confirmed by our simulations, non-resonant background increases the 
fringe visibility even if the interfering signals are generated by two different materials. Indeed, NRB from Sample is identical to that from Reference, and their interference produces high-contrast oscillations with $\Phi$. Hence, as in all CARS schemes, the proposed method requires suppressing the non-resonant signal as much as possible. This task is difficult to accomplish in multiplex CARS, where the overlap of all three excitation pulses in time is large. Ideally, one needs a long probe pulse of the duration comparable to the lifetime of the Raman coherence, but with a short front edge delayed in time with respect to the pumpStokes excitation pulses [10]. Such an optical field can be generated by propagating an ultrashort pulse through a randomly scattering medium: the sharp front edge corresponds to the ballistic part, followed by a long tail of randomly scattered photons [18].

In the final series of calculations, instead of introducing random phase modulation in the probe spectrum, we used the transmission spectrum of a random layered medium, calculated using the transfer matrix method [19]. Our simulations show that the integral interference curves of Fig.3 are well reproduced as long as the widths of well separated un-correlated lines in the probe spectrum are smaller than the differences between the Raman lines. We refer the reader to Ref.[20] for a detailed discussion of the transmission lines of a random layered medium, and of their dependance on the system parameters.

\section{EXPERIMENTAL PROCEDURE AND RESULTS}

Experimental results have been obtained using the setup shown in Fig.4. It consisted of a laser system based on a femtosecond Ti:Sapphire oscillator (Synergy, Femtolasers), a regenerative amplifier (Spitfire Pro, Spectra Physics) and an optical parametric amplifier (OPA) (Topas, Light Conversion). The amplifier generated $3 \mathrm{~mJ}, 35 \mathrm{fs}$ pulses at the central wavelength of $800 \mathrm{~nm}$ and $1 \mathrm{kHz}$ repetition rate. A portion of the $800 \mathrm{~nm}$ beam was coupled into a home-built spectral shaper based on a 640-element liquid crystal spatial light modulator (CRI, USA). The shaper produced probe pulses with a random spectral profile. The latter consisted of 25 randomly distributed Lorentzians of $1 \mathrm{~nm}$ width (full width at half maximum) and random relative phases, as required in the proposed approach. One example of such a random probe spectrum is shown in Fig.5(a). The corresponding temporal envelope of the pulse is plotted in panel (b), which demonstrates another key feature of the used pulse shape - a sharp rising edge and a long random pulse train behind it. 
Another part $(1 \mathrm{~mJ})$ of the $800 \mathrm{~nm}$ beam was used to pump an OPA, which produced Stokes and pump pulses at 1240 (signal) and $1125 \mathrm{~nm}$ (second harmonic of idler), respectively. These pulses were synchronized in time, whereas the front edge of probe pulses was delayed by 200 fs with respect to the overlapping pump-Stokes pairs. All three beams were collimated in a vertical plane (see insert in Fig.4) and focused with a $25 \mathrm{~cm}$ focal distance silver mirror into a $200 \mu \mathrm{m}$ optical path cuvette with $50 \mu \mathrm{m}$ thick walls serving as Reference.

The beams were then collimated by the second $25 \mathrm{~cm}$ focal distance silver mirror. Probe and Stokes beams, together with the generated CARS beam passed through a fixed $50 \mu \mathrm{m}$

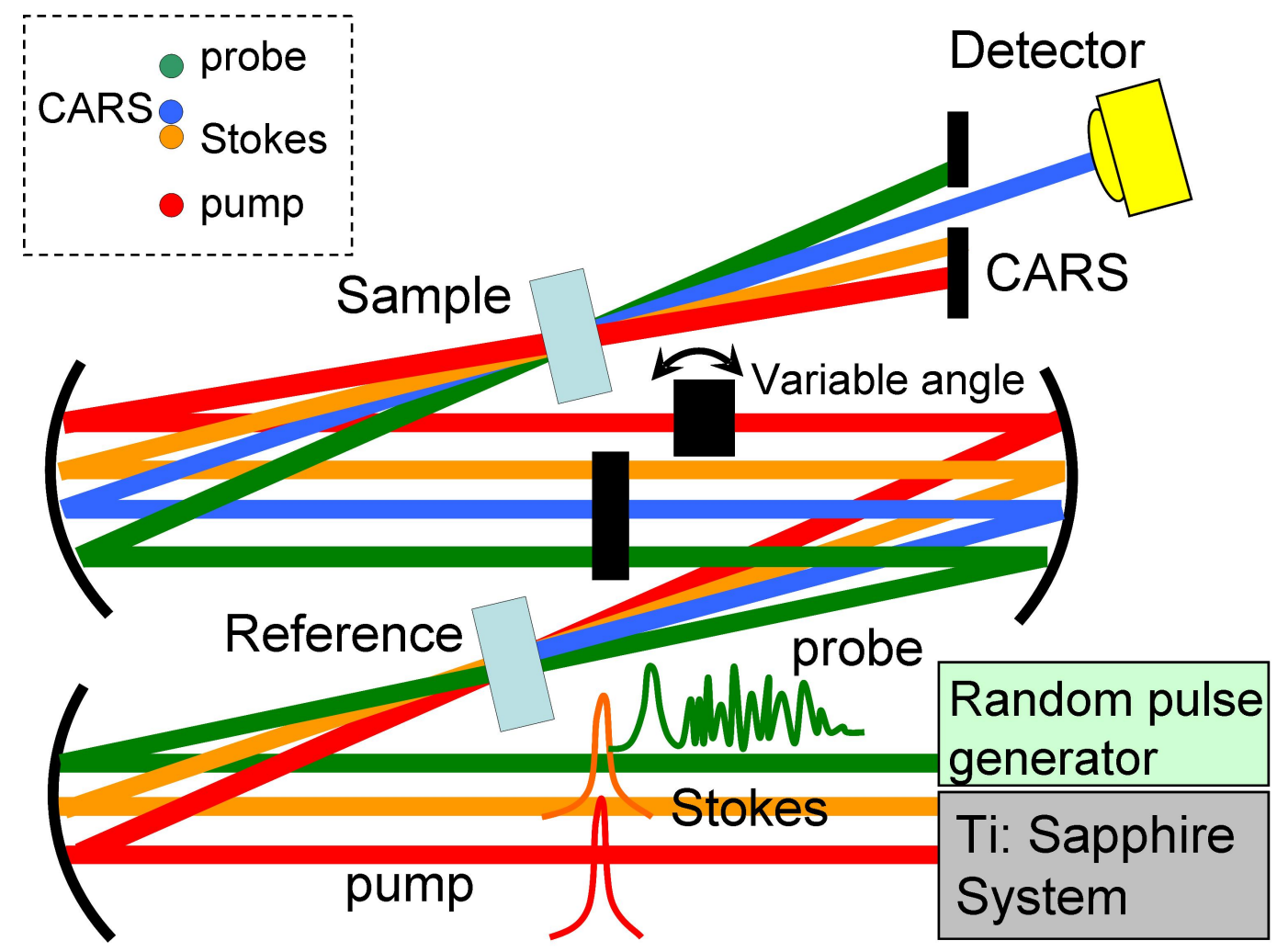

FIG. 4: Experimental setup. Femtosecond Ti:Sapphire laser system with OPA generates pump pulses at $1240 \mathrm{~nm}$ (signal) and Stokes pulses at $1125 \mathrm{~nm}$ (second harmonic of idler). Probe pulses at $800 \mathrm{~nm}$ were coupled into a spectral pulse shaper. The shaper produced probe pulses with a random spectral profile. All three beams were collimated in a vertical plane (insert) and focused into the first (Reference) cuvette. The beams were then collimated. Probe, Stokes and CARS beams passed through a fixed coverslip glass. Pump pulses, on the other hand, passed trough a similar coverslip glass mounted on a rotation stage. All beams were finally focused into the second (Sample) cuvette. CARS signal was spatially separated and coupled into a spectrometer. 
coverslip glass. Pump pulses, on the other hand, passed trough a similar $50 \mu \mathrm{m}$ coverslip glass mounted on a rotation stage. The latter provided a variable phase shift for producing the interference fringes as required by our method. All beams were finally focused into the second cuvette with another $25 \mathrm{~cm}$ focal distance silver mirror. CARS signal was spatially separated and coupled into a spectrometer (Model 2035, McPherson) operating with the spectral resolution of $0.5 \mathrm{~nm}$ and equipped with a cooled CCD camera (iDus, Andor). Exposure time was set to 0.5 seconds, and CARS spectrum was recorded as a function of the coverslip angle $\theta$. The energy of all input beams was set at $3 \mu \mathrm{J}$ per pulse. Reference cuvette
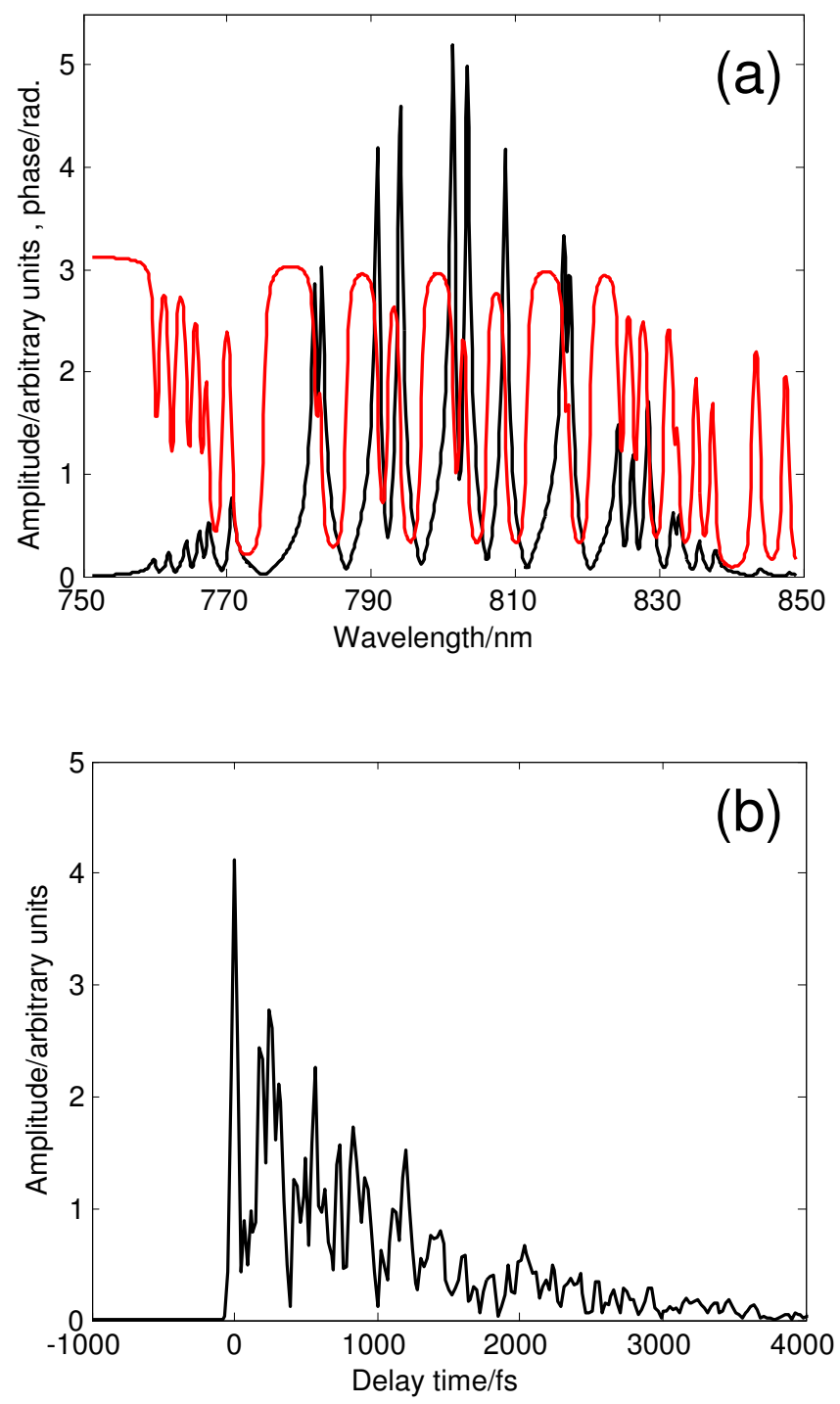

FIG. 5: An example of the probe pulse used in the experiment. a) Spectral intensity (solid black) and phase (dotted red); b) Temporal profile. 
was filled with Toluene, whereas Sample cuvette contained either Toluene or o-Xylene.

Fig.6(a,b) shows the recorded CARS interference signal for the Toluene-Toluene and Toluene-o-Xylene combinations. The pattern was averaged over five realizations of the random spectrum of probe pulses. High visibility interference fringes are clearly seen in plot (a), confirming that the two materials - Reference and Sample, are very similar in their CARS response. Unfortunately, the parabolic shape of the fringes in the $(\Phi, \lambda)$ plane did not allow us to integrate the signal over frequency while preserving the fringe contrast. We attribute the fringe curvature to chromatic dispersion, which causes an additional phase accumulation between the $S$ and $R$ media, and cannot be easily compensated. Similar pattern was observed in the interference pattern for the non-resonant signals, and reproduced in our numerical analysis. We note that in the absence of dispersion, our calculations predict that the 2D interference map for the case of " $S=R$ " is a series of vertical strips. Hence no frequency-resolved detection will be necessary to detect the fringe contrast in the absence of dispersion.

Even though integrating the two-dimensional experimental signal over $\omega$, and hence eliminating the need for a spectrometer, was not possible at this stage, we demonstrate that the fringe visibility at each wavelength is quite strong. This is seen in Fig.6(c), which shows two cross sections of the full map taken at two arbitrary values of $\lambda$ (two horizontal lines between plots (a) and (b)). In contrast, both the 2D interference map and the $1 \mathrm{D}$ cross sections for the Toluene--O-Xylene combination, shown respectively in Figs.6(b) and (d), exhibit irregular fringes with much lower visibility. This qualitative result of the proof-of-principle experiment confirms the feasibility of the proposed method.

\section{SUMMARY AND DISCUSSION}

We have shown theoretically, and demonstrated experimentally, that the interference of two CARS signals obtained by scattering noisy light from two Raman active samples offers a good measure of their chemical similarity. Provided the concentrations are similar, the interference is determined by a single parameter $w_{R S}$ (Eq.(8)), which represents the degree of overlap between the Raman lines, averaged over all pairs of lines. Even though spectrally resolved detection was required at the present stage of the experimental development, we plan to eliminate this requirement in the future work. This could be done by either compen- 

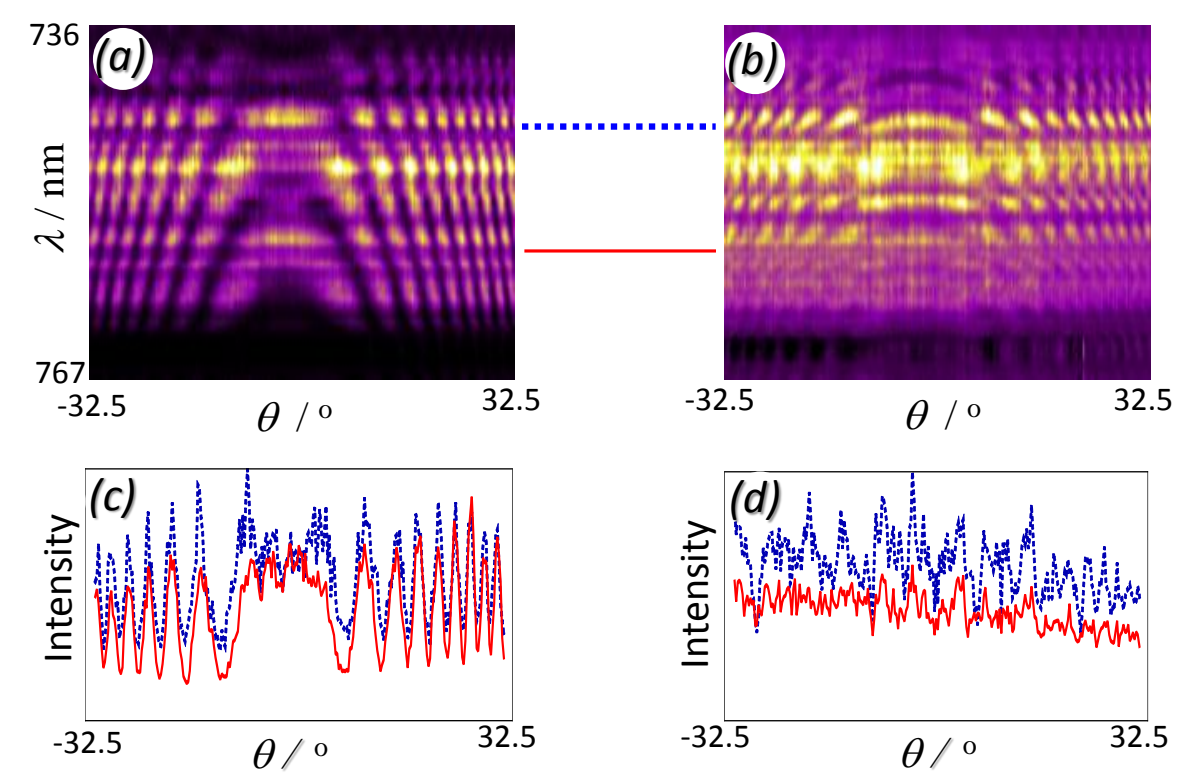

FIG. 6: Color online. Experimental results. Two-dimensional interference patterns for ToluneToluene (a) and Toluene-o-Xylene combinations (b). (c,d): Respective cross-sections of the 2D maps at two fixed wavelengths denoted by dashed blue and solid red lines. values.

sating the residual chromatic dispersion, or by filtering the detected signal with a band-pass filter, which was effectively demonstrated in this work.

For accommodating the samples of significantly different concentration, our method will have to include an additional calibration step. Using the non-resonant background, the $R$ and $S$ contributions are first equalized by attenuating one of the two CARS signals until the contrast of the non-resonant interference reaches 100\%. After that, NRB is eliminated by delaying probe pulses and the resonant interference signal is detected. Further, excitation in the fingerprint region, rather than across the whole Raman active range, can be used for increasing the detection sensitivity. Finally, the required noisy probe can be generated by sending a broadband pulse through a randomly scattering medium as discussed in the text above. With these improvements, the proposed interference method may become a powerful tool for a quick preliminary test: if the interference contrast is above a certain threshold, a more accurate frequency- or time-resolved analysis is executed.

By exploiting the interference of two noisy pulses for retrieving the information about the samples in question, our method employs a general principle known as CoherenceObservation-by-Interference-Noise ("COIN") [21-23]. In COIN, the interference of two noisy 
signals is used to deduce the degree of their coherence. In our case, the latter is equivalent to the degree of their spectral similarity. If the two spectra of interest are similar, the two signals (however noisy!) will interfere and produce fringes of high contrast. When, on the other hand, the spectra are different, the interference is suppressed and the the fringe visibility decreases. Random spectral noise has been recently exploited in coherent nonlinear spectroscopy $[20,24,25]$, and this work extends its applications to interferometric CARS techniques.

This work was supported by DTRA, CFI and NSERC.

[1] S. Roy, P. Wrzesinski, D. Pestov, T. Gunaratne, M. Dantus, J.R. Gord, Appl. Phys. Lett. 2009; 95, 074102 .

[2] J. Rehbinder, C. Pohling, T. Buckup, M. Motzkus, Opt. Lett. 2010; 35, 3721.

[3] A. Zumbusch, G.R. Holtom, X.S. Xie. Phys. Rev. Lett., 1999; 82, 4142.

[4] E.O. Potma, W.P. de Boeij, P.J.M. van Haastert, and D.A. Wiersma. PNAS 2001; 98, 1577.

[5] S.O.Konorov, X.J.G. Xu, J.W. Hepburn and V. Milner Phys. Rev. A 2009; 79, 031801.

[6] R. Lausten, O. Smirnova, B.J. Sussman, S. Grafe, A.S. Mouritzen, A. Stolow, J. Chem. Phys. 2008; $128,244310$.

[7] S.-H.Lim, A.G. Caster, and S.R. Leone Phys. Rev. A. 2005; 72, 041803(R).

[8] D. Pestov, R.K. Murawski, G.O. Ariunbold, X. Wang, M. Zhi, A.V. Sokolov, V.A. Sautenkov, Y.V. Rostovtsev, A. Dogariu, Yu Huang, M.O. Scully, Science 2007; 316, 265.

[9] Y. Silberberg, Annu. Rev. Phys. Chem. 2009; 60, 277, and references therein.

[10] S.O. Konorov, M.W. Blades, R.F.B. Turner, Appl. Spectr. 2010; 64, 767.

[11] X.J.G. Xu, S.O. Konorov, J.W. Hepburn and V. Milner, Opt. Lett. 2008; 33, 1179.

[12] S. Postma, A.C.W. van Rhijn, J.P. Korterik, P. Gross, J.L. Herek and H.L. Offerhaus, Opt. Express 2008; 16, 7985.

[13] D. Oron, N. Dudovich and Y. Silberberg, Phys. Rev. Lett. 2003; 90, 213902.

[14] B. Xu, J.M. Gunn, J.M. Dela Cruz, V.V. Lozovoy and M. Dantus. J. Opt. Soc. Am. B 2006; 23,750 .

[15] N. Dudovich, D. Oron and Y. Silberberg, J. Chem. Phys. 2003; 118, 9208.

[16] H. Okamoto, Chem. Phys. 1991; 155, 291. 
[17] V.F. Kamalov, Y.P. Svirko, Chem. Phys. Lett. 1992; 194, 13.

[18] P.M. Johnson, A. Imhof, B.P.J. Bret, J. Gomez Rivas, and A. Lagendijk, Phys. Rev. E 2003; 2003, 016604.

[19] J.B. Pendry, Adv. Phys. 1994; 43, 461.

[20] T.M. Drane, J.W. Hepburn, V. Milner, J. Mod. Optics 2010; 57, 1928.

[21] O. Kinrot, I.Sh. Averbukh, Y. Prior, Phys. Rev. Lett. 1995; 75, 3822.

[22] C. Leichtle, W.P. Schleich, I.Sh. Averbukh, M. Shapiro J. Chem. Phys. 1998; 108. 6057.

[23] Ch. Warmuth et. al., J. Chem. Phys. 2001; 114, 9901.

[24] X.G. Xu, S.O. Konorov, J.W. Hepburn, V. Milner, Nature Physics 2008; 4, 125.

[25] S.O. Konorov, X.G. Xu, J.W. Hepburn, V. Milner, Phys. Rev. A 2009; 79, 031801(R). 